\title{
Ultra-fine grinding of inorganic non-metallic materials using various types of bonds and processing strategies
}

\author{
Christian Schulze ${ }^{1, *}$, Sebastian Henkel $^{1}$, and Jens Bliedtner ${ }^{1}$ \\ ${ }^{1}$ Ernst-Abbe University of Applied Sciences Jena, Carl-Zeiss-Promenade 2, 07745 Jena, Germany
}

\begin{abstract}
Ultra-fine grinding is a technology, which can be used to process inorganic non-metallic materials. High quality surfaces with a low roughness, and high flatness are achievable in a low processing time. The experimental setup which is described shall compare face grinding with lateral grinding in a single processing step using various materials, bonds and processing parameters. The results are supposed to deepen the knowledge about this grinding technology to further improve the properties of fabricated components.
\end{abstract}

\section{Ultra-fine grinding}

Due to the developments of mankind in regard to machining materials, such as using high accurate CNC machines in collaboration with advancing tool technologies, high quality surfaces can be achieved. Ultra-fine grinding can be considered as part of precision grinding with low removal rates using tools with a small diamond grain size as shown in Fig. 1. [1]

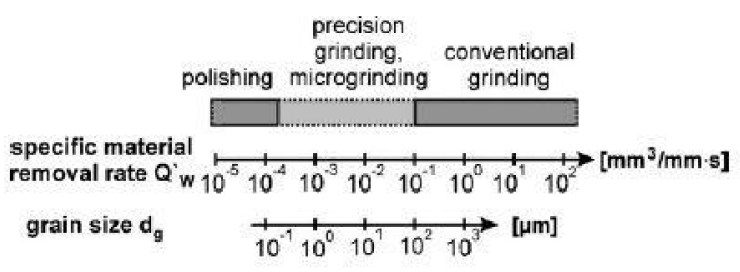

Fig. 1. Properties of grinding [1]

Especially when processing glass, high quality surfaces with a low roughness, high flatness and low processing times are demanded. Two strategies of grinding to fulfil those demands are face grinding and lateral grinding. [2] The operating principles are displayed in Fig. 2.

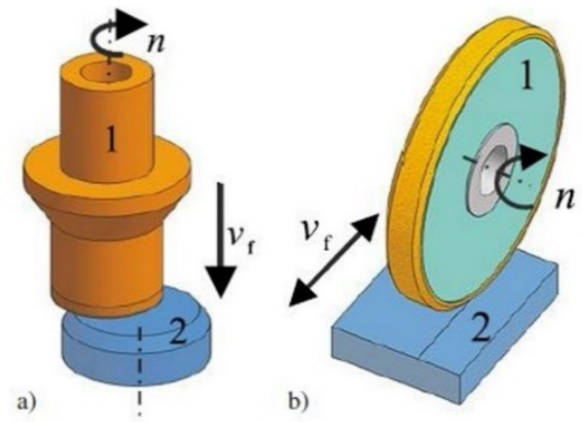

Fig. 2. Grinding strategies: a) face grinding b) lateral grinding 1: tool 2: sample [2]

\section{Experimental setup}

\subsection{Description}

The conducted experiments are based on former experiments and shall reveal the differences in lateral grinding in comparison with face grinding as well as a combination of lateral and face grinding in a single processing step, in regards to achievable surface roughness, occurring process forces as well as subsurface damages. In order to realise this experiment, a special sample geometry was designed and prepared, as shown in Fig. 3.

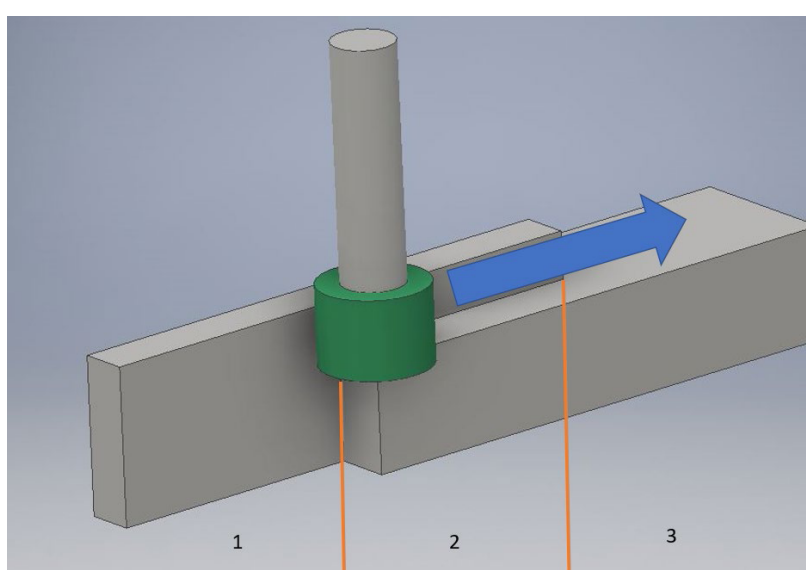

Fig. 3. Sample geometry and tool kinematic.

The samples used are $20 \times 20 \times 100 \mathrm{~mm}^{3}$ in size. The tool will move in a straight line, processing 3 different areas. The first area will be lateral grinding only (1), the second area will be a combination of face and lateral grinding (2) and the third area will be face grinding only (3) as marked in Fig. 3. Due to the geometry of the sample as well as the tool, the cutting speed will have the same value for face grinding as well as lateral grinding.

\footnotetext{
* Corresponding author: christian.schulze@eah-jena.de
} 


\subsection{Parameters}

A 5-axis CNC machine from company SAUER (DMG) will be used to conduct the experiments as pictured in Fig. 4.

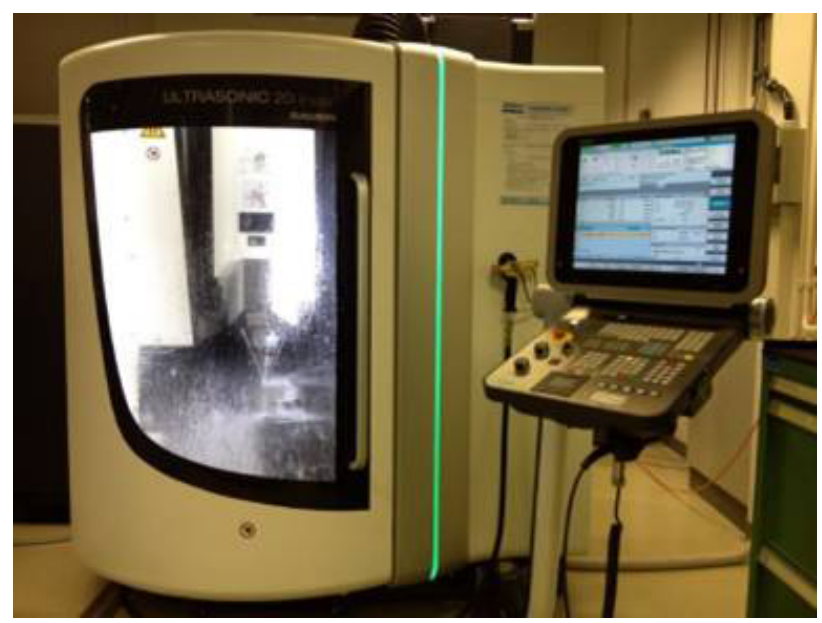

Fig. 4. CNC grinding machine: Ultrasonic 20 linear.

Besides enabling a rotational speed up to $42000 \mathrm{rpm}$, an ultrasonic assistance is integrated in this system. It can induce a high-frequency oscillation (about $20-30 \mathrm{kHz}$ ) of a few $\mu \mathrm{m}$ alongside the $\mathrm{z}$-axis of the machine in addition to the tool rotation. This results in numerous benefits: possible reduction of roughness and subsurface damages, wear reduction of the tool as well as force reduction. [3]

The process influence of the following parameters will be investigated:

- 2 materials: fused silica, BK7

- 2 diamond tools, $\varnothing 14 \mathrm{~mm}$ D16: metal bond, resin bond

- values of cutting depth

- values of cutting width

- values of cutting speed

- values of feed rate

- state of ultrasonic assistance (on, off)

The following parameters will be examined:

- roughness

- waviness

- subsurface damages

- process forces

\section{Perspective}

Ultra-fine grinding processes combined with modern tool technologies have proved themselves to significantly improve the quality of glass surfaces in already conducted experiments. As a result, transparent, reflective surfaces are achievable with a roughness improvement up to $R a=8 \mathrm{~nm}$ and $R q=10 \mathrm{~nm}$ by face grinding fused silica with a resin bonded tool as shown in Fig. 5. [4]

This results in a significant reduction of polishing time needed in following processes, reducing the necessary total processing time about $40 \%$ (Fig. 6). [4]

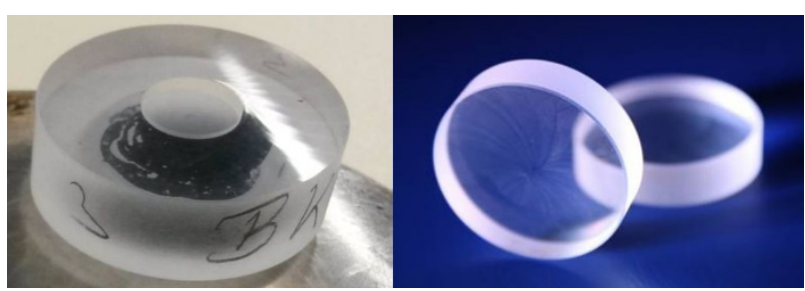

Fig. 5. Achieved surface qualities by ultra-fine face grinding

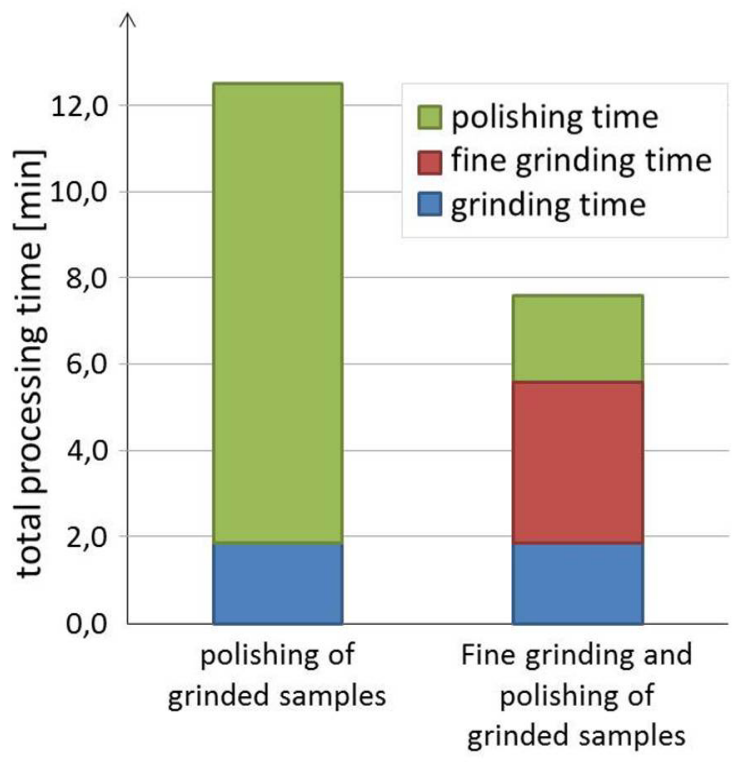

Fig. 6. Comparison of polishing time of fused silica samples by fine grinding using a resin bonded tool and samples grinded with a metallic bond D35 Tool. [4]

Therefore, more investigations are necessary in order to further improve the technology of ultra-fine grinding in terms of achievable surface quality as well as its economic aspects. The experiments focusing lateral and face ultrafine grinding are currently in preparation. First results are soon to be expected.

\section{References}

1. E. Brinkmeier, Y. Mutlugünes, F. Klocke, J.C. Aurich, P. Shore, H. Ohmori : Ultra-precision grinding. CIRP Annals, Manufacturing Technology. Vol. 59, Page 652-671. (2010)

2. J. Bliedtner, G. Gräfe, R. Hector : Optical technology. McGraw-Hill Education. (2011)

3. J. Bliedtner, S. Henkel, A.-S. Schwager, E. Rädlein, M. Gerhardt: Ultrafine grinding and laser beam polishing processes for the manufacturing of freeform optics. Delft (NL): EOSAM 2018, Presentation. (2018)

4. S. Henkel, J. Bliedtner, E. Rädlein, C. Schulze, M. Rost, M. Gerhardt, M. Fuhr : Ultra-fine grinding of silicate materials under the use of new resin bond diamond tools. Proceedings of SPIE Vol. 10326, Teisnach: 4th European Seminar on Precision Optics Manufacturing, 10326 05. (2017) 